\title{
Design of telecentric imaging systems for noncontact velocity sensors
}

\author{
Christian Berger \\ Universität Karlsruhe (TH) \\ Institut für Mess- und Regelungstechnik \\ 76131 Karlsruhe, Germany \\ E-mail: Christian.Berger@mrt.uka.de
}

\begin{abstract}
Optical sensors for noncontact velocity measurement require imaging systems with constant optical magnification in spite of the variable distance between the sensor and the object of measurement. This requirement can be met using telecentric systems. When such systems are set up, several design considerations must be taken into account. These concern the decision as to whether a single or a double telecentric system shall be used, as well as the choice of the optical magnification and the dimensioning of the aperture stop. The results of the theoretical analysis are confirmed by experiments with a prototype velocity sensor for belt drives. (c) 2002 Society of Photo-Optical Instrumentation Engineers. [DOI: 10.1117/1.1504105]
\end{abstract}

Subject terms: noncontact velocimetry; correlation sensor; telecentric imaging; magnification error; luminous intensity.

Paper 010322 received Sep. 6, 2001; revised manuscript received Mar. 26, 2002; accepted for publication Mar. 26, 2002.

\section{Introduction}

Determining the velocity of technical surfaces as, e.g., paper webs, ${ }^{1}$ textile yarns, ${ }^{2}$ or V-belts ${ }^{3-5}$ is a frequent measurement task. To achieve this without physically contacting the object of measurement, optical sensors are commonly used. The imaging systems of such sensors usually must ensure a constant optical magnification at a variable object distance from the sensor. This avoids biased measurements resulting from transversely vibrating objects or positioning inaccuracy of the sensor with respect to the object surface. Telecentric systems satisfy this requirement and are relatively easy to set up for prototype sensors. Nevertheless their parameters, such as optical magnification or aperture dimensions, must be designed with care, especially if high measurement accuracy is required.

In this paper, design specifications are derived from a geometrical optical analysis of telecentric systems. Lens and aperture dimensions are assumed to be sufficiently large, hence diffraction effects can be neglected. For the sake of simplicity, aberration-free thin lenses are presumed.

\section{Noncontact Velocity Measurement with Optical Sensors}

The velocity of moving surfaces can be measured without contact using optical sensors, whose working principles are based on, e.g., correlation ${ }^{6,7}$ or spatial filter ${ }^{8,9}$ methods. Typically, such sensors have an optical system to project the object surface onto several detectors aligned in the direction of motion in its image plane. Velocity information can be obtained by evaluating the time-dependent detector signals, provided that some geometry parameters are given.

Correlation sensors receive signals from two surface segments using two detectors aligned at a distance $L^{\prime}$ [Fig. 1(a)]. In the ideal case, these signals are identical, except for a shift by a transit time $T$. Taking into account an optical magnification $M$ of the sensor's imaging system, the measured velocity is calculated from

$v_{m}=\frac{L^{\prime}}{M T}=\frac{L}{T}$

Hence, estimation of the velocity requires measuring the time lag $T$, which can be performed by calculating the cross-correlation function of the two sensor signals. ${ }^{10}$

A spatial filter sensor, in contrast, has a grating structure [Fig. 1(b)]. This leads to a periodic time-dependent sensor signal, from which the velocity is obtained by evaluating its temporal frequency $f_{t}$. Assuming a grating realized by an array of detectors with period $g^{\prime}$ in the image plane of the sensor, the signal frequency and the measured velocity are related by

$v_{m}=\frac{f_{t} g^{\prime}}{M}=f_{t} g$.

As we can see from Eqs. (1) and (2), determining the velocity from measured transit time or frequency values requires knowledge of the detector geometry parameter $L^{\prime}$ or $g^{\prime}$, and of the optical magnification $M$. These parameters must be constant to avoid systematic errors of the measured values $T$ or $f_{t}$. While fixed detector geometries obviously satisfy this requirement for $L^{\prime}$ or $g^{\prime}$, a constant optical magnification is not self-evident, considering that the distance of the sensor to the surface may vary, e.g., due to transversal surface vibrations. Thus, simple imaging systems, whose optical magnification depends on the object distance, are unsuitable for velocity sensors. One possibility to meet the special requirement of distance-independent magnification is to use telecentric imaging systems. This is investigated in detail here.

EVA-STAR (Elektronisches Volltextarchiv - Scientific Articles Repository) http://digbib.ubka.uni-karlsruhe.de/volltexte/15992002

Opt. Eng. 41(10) 2599-2606 (October 2002)

$0091-3286 / 2002 / \$ 15.00 \quad$ (c) 2002 Society of Photo-Optical Instrumentation Engineers 


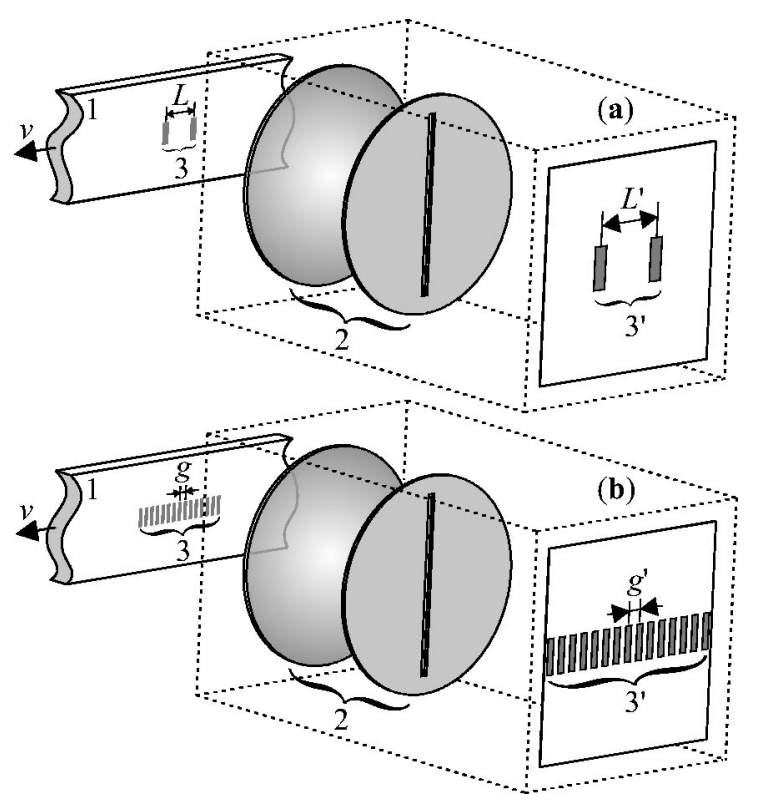

Fig. 1 Schematic setup of (a) correlation and (b) spatial filter velocity sensors: 1 : object of measurement; 2 : imaging system; 3 : surface segments evaluated by the detectors $\left(3^{\prime}\right)$.

\section{Telecentric Imaging Systems}

Telecentric systems ${ }^{11,12}$ have an aperture stop in the back focal plane of a lens. This ensures that only those bundles of rays contribute to imaging, whose chief rays are parallel to the optical axis in the object space. A single telecentric system can be built using just one lens [see Fig. 2(a)]. Likewise a system consisting of two lenses with the aperture stop in their common focal plane is referred to as a double telecentric system [see Fig. 2(b)]. In the latter system, the chief rays are parallel to the optical axis not only in the object space, but also in the image space, thus ensuring an

(a)

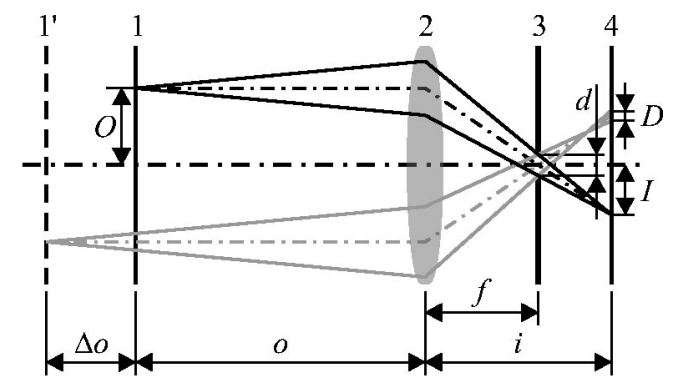

(b)

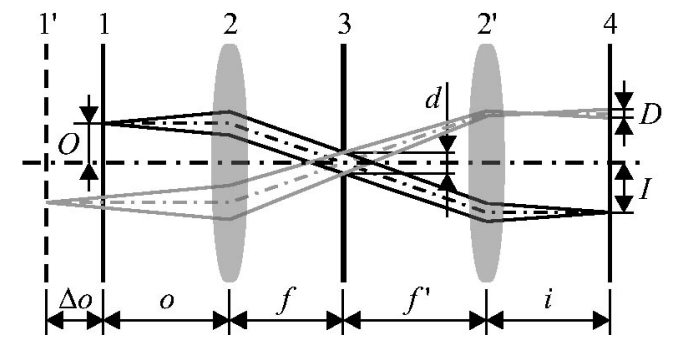

Fig. 2 (a) Single telecentric and (b) double telecentric imaging systems: 1 and $1^{\prime}$ : object plane (in and out of focus); 2 and $2^{\prime}$ : imaging lenses; 3: aperture stop; 4: image plane. orthogonal angle of incidence on the image plane. This is advantageous in sensors collecting radiation by light waveguides with small numerical apertures.

Provided that the image distance $i$ is constant, the parallel chief rays in the object space ensure that the optical magnification $M$ is invariant to the object distance. For a single telecentric system with a lens of focal length $f$ and with nominal object distance $o, M$ is given by

$M=(i-f) / f=i / o=I / O$,

where $O$ and $I$ denote object and image heights, respectively. For a double telecentric system with focal lengths $f$ and $f^{\prime}$, the following equations hold ${ }^{13}$ :

$M=f^{\prime} / f=I / O$,

$o-f=\left(f^{\prime}-i\right)\left(f / f^{\prime}\right)^{2}$.

If the object is not located at the nominal distance $o$ from the lens, but at a distance $o+\Delta o$, each object point is imaged onto a finitely small spot, i.e., the resulting image becomes blurred. The shape and dimensions of the spot are determined by the aperture stop. This can be used to design the aperture dimensions, as shown in the following.

\subsection{Design of the Aperture Dimensions}

Assuming a circular aperture of diameter $d$ in a telecentric system, every object point is projected onto a spot of diameter

$D=d M|\Delta o| / f$

(cf. Fig. 2). In terms of Fourier optics, the imaging can hence be described by a circular impulse response. Neglecting diffraction effects, the modulus of its 2-D Fourier transform is referred to as the modulation transfer function ${ }^{14}$ (MTF). This function relates the spatial frequency spectrum of the image $S_{i}$ to that of the object $S_{o}$ :

$\left|S_{i}(\mathbf{f} / M)\right|=\kappa \operatorname{MTF}(\mathbf{f} / M)\left|S_{o}(\mathbf{f})\right|$,

where $\mathbf{f}=\left(f_{x}, f_{y}\right)^{T}$ is the vector of spatial frequency coordinates, and $\kappa=$ const.

In the case of a circular impulse response of diameter $D$, the normalized MTF can be derived as ${ }^{15,16}$

$\operatorname{MTF}(\mathbf{f})=\left|\frac{2 J_{1}(\pi D|\mathbf{f}|)}{\pi D|\mathbf{f}|}\right|$.

Here $J_{1}$ denotes a Bessel function of first kind and first order, which is a spatial lowpass, as shown in Fig. 3. As the object spectrum is filtered by $\operatorname{MTF}(\mathbf{f} / M)$ according to Eq. (6), the design of the aperture diameter can proceed as follows:

1. The spatial bandwidth $\mathbf{f}_{\mathrm{bw}}$ of the object surface must be measured.

2. The maximum deviation $\left|\Delta o_{\max }\right|$ of the object from its nominal distance to the sensor must be estimated. 

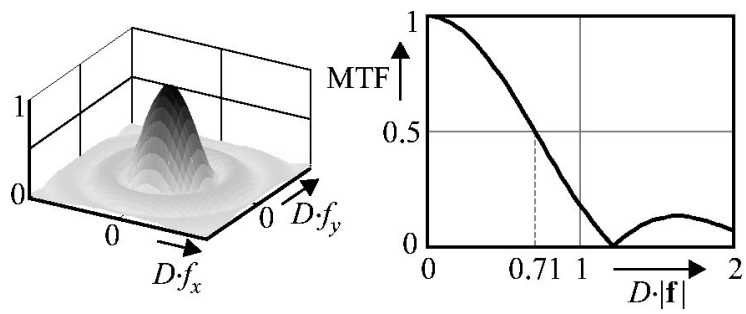

Fig. 3 MTF of out-of-focus imaging with a circular aperture.

3. A maximum allowable reduction of the finest object structures by the spatial lowpass, i.e., a minimum value $\operatorname{MTF}_{\text {min }}\left(\mathbf{f}_{\mathrm{bw}} / M\right)$ must be determined.

As an example, $\mathrm{MTF}_{\min }=0.5=: 2 J_{1}(\pi k) /(\pi k)$ is requested. The corresponding argument $k=0.71$ can be found from tables. ${ }^{15}$ Combining the considerations given with Eq. (5) leads to the following dimensioning of $d$ :

$d=\frac{0.71 f}{\left|\Delta o_{\text {max }}\right| \mathbf{f}_{\mathrm{bw}} \mid}$.

Noncircular apertures can be dimensioned accordingly. The only difference is that the MTF must be adapted to the aperture shape.

As we can conclude from Eq. (8), systems with the smallest possible aperture dimensions are highly tolerant toward variations of the object distance. However, the aperture size cannot be reduced arbitrarily, because this would strongly reduce the luminous intensity of the image.

\subsubsection{Luminous intensity}

It can be understood intuitively that the amount of light passing through an optical system decreases when the opening of the aperture is reduced. The luminous intensity in the image plane and the energy of the time-dependent sensor signals hence decrease accordingly. To a certain degree, this loss of signal energy can be compensated for. In time-continuous systems, e.g., sensors with photodiodes, the gain of the signal processing unit can be increased; in time-discrete systems such as CCD sensors, ${ }^{17}$ one could alternatively increase the integration time. However, these compensations are not applicable to correct arbitrarily weak image intensities. Electronic circuits with large gains tend to produce a high noise level or may even have stability problems, whereas long CCD integration times reduce the measurement dynamics. As a result, telecentric systems of optical sensors should be designed to allow for a highest possible luminous intensity in the image plane.

In the following, the influence of the aperture dimensions on the luminous intensity is investigated quantitatively. The object surface is assumed to reflect the illuminating radiation diffusely, so Lambert's cosine law applies. ${ }^{18}$ The energy emerging from a surface element $\Delta S$ onto an element $\mathrm{d} \Omega$ of the solid angle is then given by ${ }^{19}$

$\mathrm{d} E=B \Delta S \cos \sigma \mathrm{d} \Omega=B / 2 \Delta S \mathrm{~d} \varphi \mathrm{d}\left(\sin ^{2} \sigma\right)$,

where $B=$ const. denotes the photometric brightness of $\Delta S$, $\varphi$ is the azimuth angle of $\mathrm{d} \Omega$, and $\sigma$ is the angle between

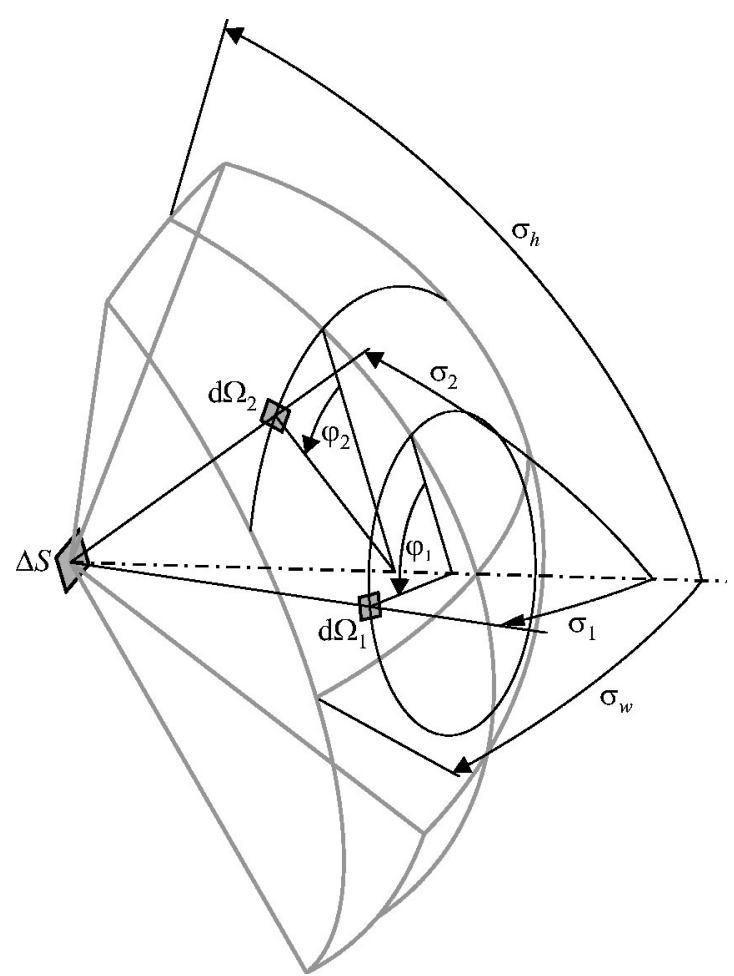

Fig. 4 Illustration of a surface element $\Delta S$ radiating into elements $\mathrm{d} \Omega$ of the solid angle.

the normal vectors of $\Delta S$ and $\mathrm{d} \Omega$ (see Fig. 4). From this, the total energy radiated from $\Delta S$ into the half-space in front of it is obtained as

$E_{t}=B / 2 \Delta S \int_{0}^{\sin ^{2} \pi / 2} \int_{0}^{2 \pi} \mathrm{d} \varphi \mathrm{d}\left(\sin ^{2} \sigma\right)=\pi B \Delta S$.

Now $\Delta S$ is assumed to be a surface element in front of a telecentric system. It is further assumed that $\Delta S$ is near the optical axis of the system, so that the solid angle of the imaged radiation is not limited by the edge of the imaging lens. Hence, for systems with circular aperture stops, the radiation within a spherical cone limited by an angle $\sigma_{l}$ $=\arctan d /(2 f)$ (cf. Fig. 2) is imaged by the system. Thus, only a portion

$\frac{E}{E_{t}}=\sin ^{2} \sigma_{l}=\sin ^{2}\left(\arctan \frac{d}{2 f}\right)$

of the total energy radiated from $\Delta S$ is reaching the image plane. As we can see in Fig. 5(a), $E / E_{t}$ is strongly decreasing in systems with low ratios $d / f$.

For the particular application of 1-D velocity measurement, an elegant solution to this problem can be found. The optical system does not necessarily have to be ideally telecentric in that case, but it must ensure a distanceindependent optical magnification only in the direction of object motion. Thus, rectangular instead of circular aperture stops can be used to widen the opening of the system, ${ }^{20}$ provided that the short axis of the aperture slit is parallel to the object velocity (cf. Fig. 1). For a slit of width $w$ and 
(a)

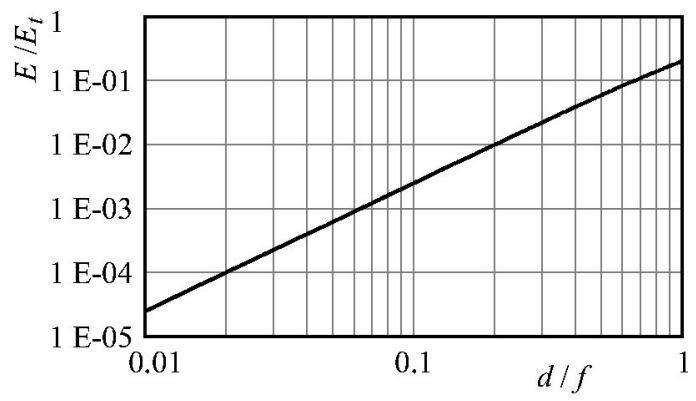

(b)

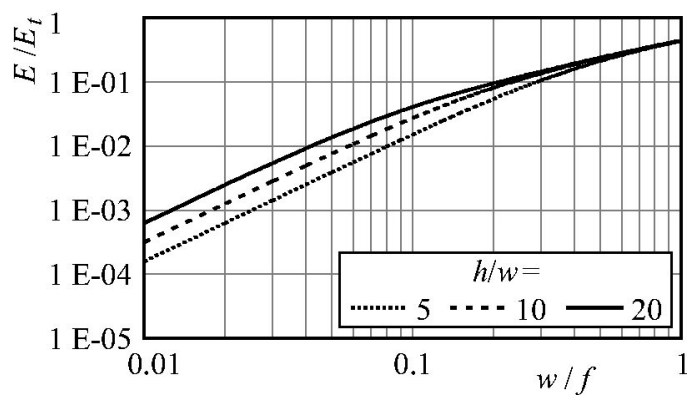

Fig. 5 Portion of the total energy reaching the image plane of telecentric systems with (a) circular and (b) rectangular aperture stops.

height $h>w$, the radiation falling onto a part of the solid angle limited by angles $\sigma_{w}=\arctan w /(2 f)$ and $\sigma_{h}$ $=\arctan h /(2 f)$ (cf. Fig. 4) is reaching the image plane. The corresponding energy can be derived as

$\frac{E}{E_{t}}=\sin ^{2} \sigma_{w}+\frac{2}{\pi} \int_{\sin ^{2} \sigma_{w}}^{\sin ^{2} \sigma_{h}} \arcsin \left[\left(\frac{1-a}{a}\right)^{1 / 2} \tan \sigma_{w}\right] \mathrm{d} a$.

Evaluating Eq. (12) by numerical integration yields the curves of Fig. 5(b). When these are compared to the values of Fig. 5(a), one notices that the energy in the image plane is about one order of magnitude higher in systems with slits than with circular aperture stops, provided that systems with common ratios $d / f<0.1$ or $w / f<0.1$ are considered.

As an example, telecentric systems (single or double) with $f=20 \mathrm{~mm}$ and with circular or rectangular apertures are compared. The normalized energies in the image plane are calculated with Eqs. (11) and (12). The results (see Table 1) show that the gain of signal energy using a rectangular instead of a circular aperture can reach factors of up to 25 in this example.

Table 1 Comparison of normalized image plane energies in telecentric systems with $f=20 \mathrm{~mm}$.

\begin{tabular}{lcc}
\hline \hline Aperture & Dimensions & $E / E_{t}$ \\
\hline Circular & $0.4 \mathrm{~mm}$ & $1.0 \times 10^{-4}$ \\
& $0.4 \mathrm{~mm} \times 2 \mathrm{~mm}$ & $6.3 \times 10^{-4}$ \\
Rectangular & $0.4 \mathrm{~mm} \times 4 \mathrm{~mm}$ & $1.3 \times 10^{-3}$ \\
& $0.4 \mathrm{~mm} \times 8 \mathrm{~mm}$ & $2.5 \times 10^{-3}$ \\
\hline \hline
\end{tabular}

(a)

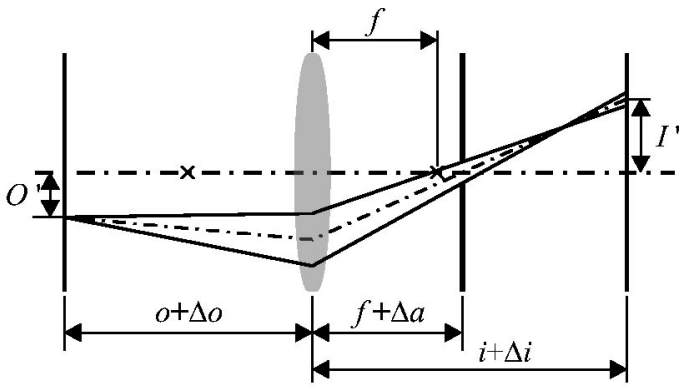

(b)

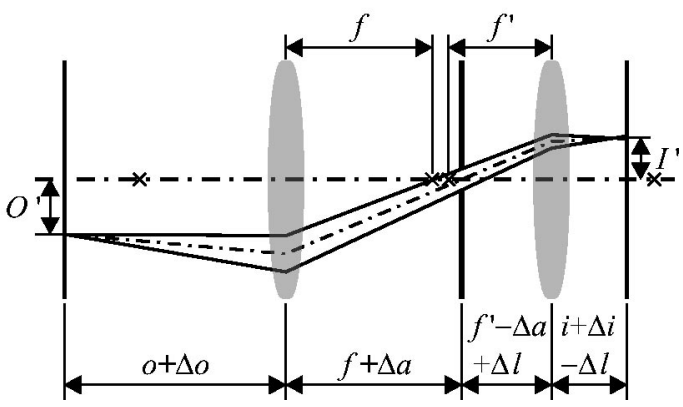

Fig. 6 (a) Single and (b) double telecentric imaging systems with axial positioning errors of their optical elements.

\subsection{Impact of Inaccurate Axial Adjustment of the Optical Elements}

A constant optical magnification $M$ applies only to those systems where the telecentric aperture stop is positioned exactly within the focal plane of one or two lenses (cf. Fig. 2 ). This requirement is not necessarily satisfied. The adjustment of an optical system could deviate from the optimum setup, e.g., due to thermal expansion. Moreover, the focal length of simple lenses is usually specified within a tolerance of $\pm 2 \%$ of its nominal value; this can be another reason for inaccurate axial positioning of the aperture relative to the lens.

In the following, optical imaging with inaccurately adjusted telecentric systems is investigated. The axial positioning errors of the aperture stop and of the image plane are denoted by $\Delta a$ and $\Delta i$, respectively. Furthermore, in the case of a double telecentric system, an axial misalignment $\Delta l$ of the second imaging lens is assumed.

As we can see in Fig. 6, the chief rays of the optical imaging are not parallel to the optical axis in the object space of such systems. Hence, the optical magnification $M^{\prime}=I^{\prime} / O^{\prime}$ is no longer constant, but depends on the object distance $o+\Delta o$. To investigate the sensitivity to axial misalignments, the relative deviation $\Delta M / M=\left(M^{\prime}-M\right) / M$ is calculated by analyzing the ray traces of the systems shown in Fig. 6. For single telecentric systems, a relative magnification error of

$$
\frac{\Delta M}{M}=\frac{1+(1+M) /(M-\Delta a / f) \Delta i / i}{1-(1+M) /(M-\Delta a / f) \Delta a / f \Delta o / o}-1
$$

can be found. For double telecentric systems, a more complex formula is obtained: 

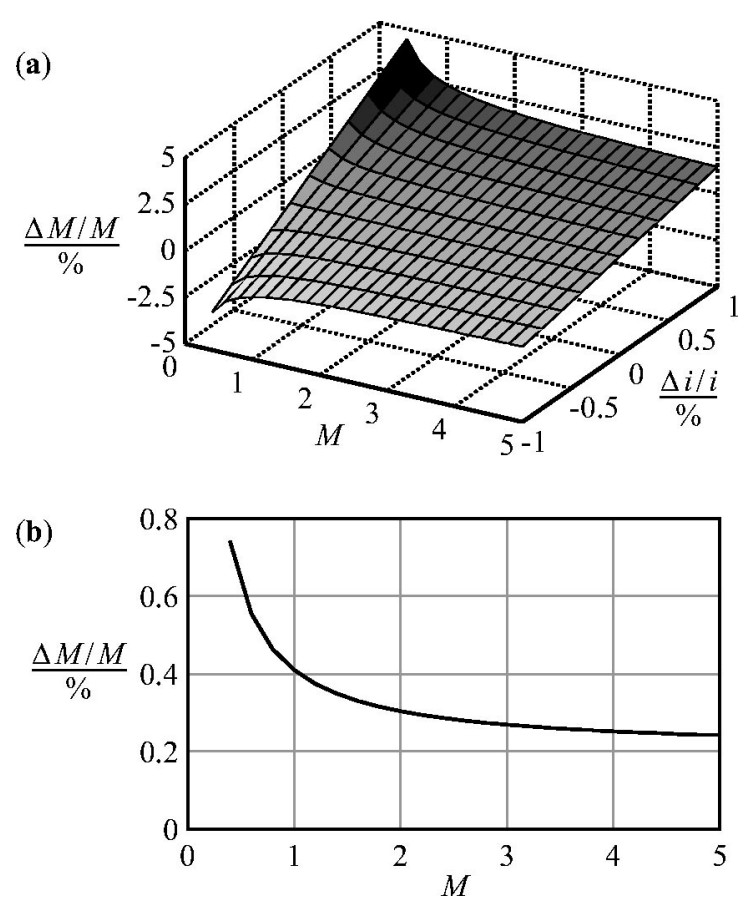

Fig. 7 Single telecentric system, $\Delta o / o=10 \%, \Delta a / f=2 \%$ : relative error of the optical magnification for (a) $|\Delta i / i| \leqslant 1 \%$ and (b) $\Delta i=0$.

$$
\begin{aligned}
\frac{\Delta M}{M}= & \left\{1-\frac{\Delta a}{f}\left[\frac{o}{f}\left(1+\frac{\Delta o}{o}\right)-1\right]\right\}^{-1}\left(1+\frac{\Delta a}{f}\left(1-\frac{o}{f}\right)\right. \\
& +\frac{\Delta l}{f^{\prime}}\left\{\frac{\Delta l}{f^{\prime}}-M\left(1-\frac{o}{f}\right)-\frac{\Delta i}{i}\left[M\left(1-\frac{o}{f}\right)+1\right]\right. \\
& \left.\left.-\frac{1}{M} \frac{\Delta a}{f}\right\}+\frac{\Delta i}{i} \frac{\Delta a}{f}\left(1-\frac{o}{f}+\frac{1}{M}\right)\right)-1 .
\end{aligned}
$$

As we can conclude from Eq. (13), the relative magnification error of single telecentric systems is influenced by the relative axial misalignment $\Delta i / i$ of the image plane in a term of first order. In contrast, Eq. (14) shows that for double telecentric systems, $\Delta M / M$ depends on relative positioning errors only in terms of second order, provided that $o / f=1$. This is an important result. It implies that the nominal object and image distances should equal the focal lengths of the double telecentric system's lenses [cf. Eq. (4)] to obtain a minimum sensitivity to axial positioning errors of the optical elements.

To show the difference between single and double telecentric systems, relative deviations of $|\Delta i / i| \leqslant 1 \%$ and $\Delta a / f=2 \%$ and an object distance deviation of $\Delta o / o$ $=10 \%$ are assumed.

According to the considerations just given, the double telecentric system is designed with $o=f$ and $i=f^{\prime}$; the relative positioning error of the second lens is assumed to be $\Delta l / f^{\prime}=-2 \%$. Insertion of these values in Eqs. (13) and (14) yields Figs. 7(a) and 8(a). These figures show first that the deviations $\Delta M / M$ are significantly lower for double than for single telecentric systems, i.e., double telecentric systems are less sensitive to axial positioning errors of their
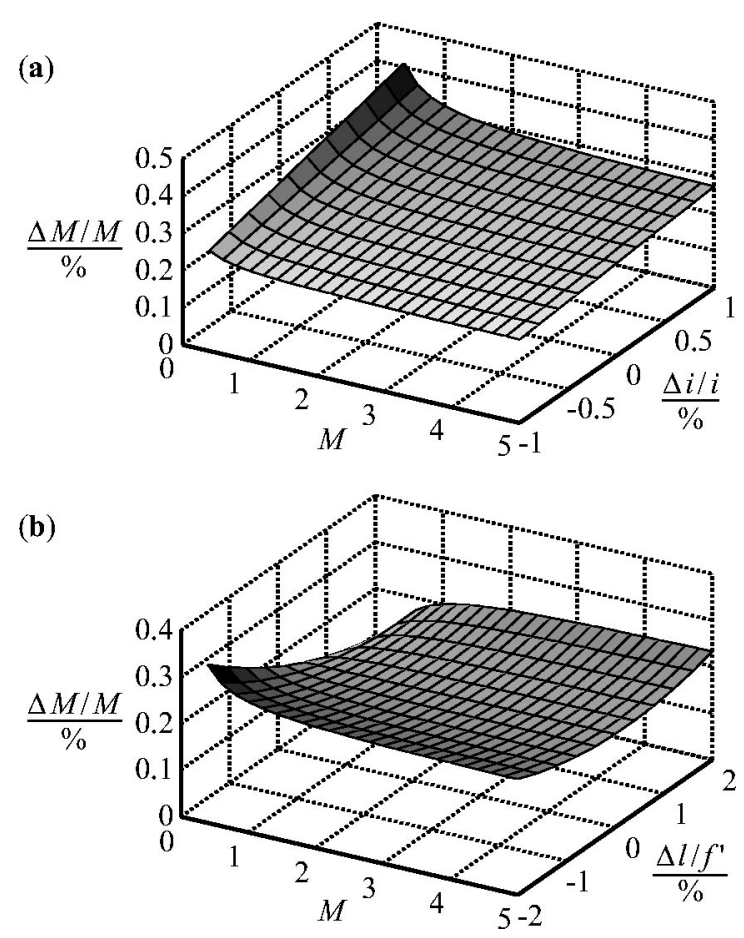

Fig. 8 Double telecentric system, $o=f, \Delta o / o=10 \%, \Delta a / f=2 \%$ : relative error of the optical magnification (a) for $|\Delta i / i| \leqslant 1 \%$ and $\Delta / / f^{\prime}=-2 \%$ and (b) for $\Delta i=0$ and $\left|\Delta / / f^{\prime}\right| \leqslant 2 \%$.

optical elements. Moreover, we can see that for any given $\Delta i / i$, the highest errors $\Delta M / M$ occur at small $M$ values. Hence, the design of telecentric systems with small optical magnifications of $M<1$ should be avoided.

Assuming a perfectly adjusted image plane, i.e., $\Delta i$ $=0$, the Figs. 7(b) and 8(b) are obtained. Despite the fact that the single telecentric system is no longer influenced by first-order error terms [cf. Eq. (13)], the relative deviations $\Delta M / M$ are still higher than for double telecentric systems, provided that systems with small optical magnifications $M$ are compared. For high $M$ values, the errors $\Delta M / M$ of single and double telecentric systems are of the same order of magnitude. The double telecentric system could be disadvantageous only if large positioning errors $\Delta l / f^{\prime}$ of the second imaging lens occurred.

\section{Experimental Results}

Based on the preceding considerations, a new sensor was designed for the application of slip measurement in vehicle belt drives. The velocity of the V-belt is evaluated by correlation techniques and compared to rotational velocities of belt pulleys, which are obtained from ordinary revolution counters.

The optical setup of the sensor is shown in Fig. 9. It is a double telecentric system with focal lengths of $f=20 \mathrm{~mm}$ and $f^{\prime}=30 \mathrm{~mm}$, from which results a nominal optical magnification of $M=1.5$ [cf. Eq. (4)]. According to the considerations given in Sec. 3.2, the image distance and the nominal object distance equal the focal lengths of the system, i.e., $i=30 \mathrm{~mm}$ and $o=20 \mathrm{~mm}$. The telecentric aperture is rectangular with slit dimensions of $w=0.4 \mathrm{~mm}$ and $h$ 


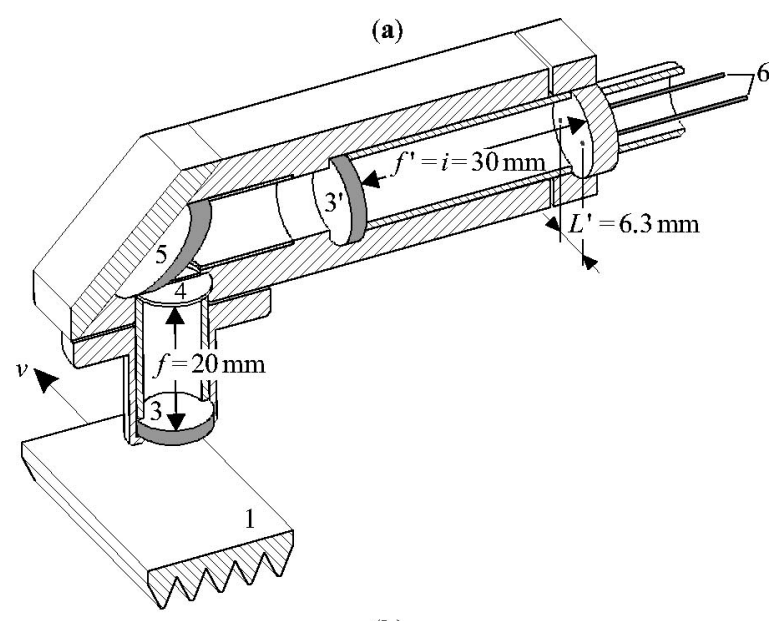

(b)

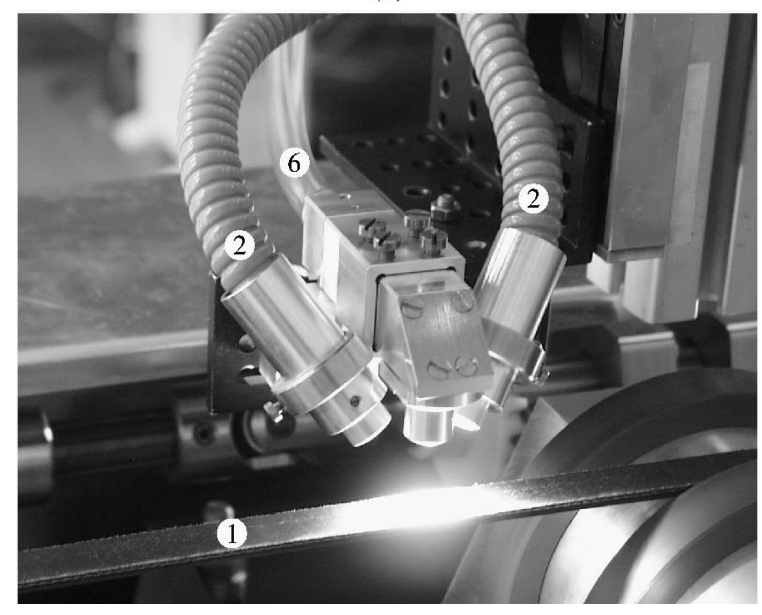

Fig. 9 (a) Mechanical setup and (b) photography of the new velocity sensor with double telecentric optical system: 1: V-belt; 2: illumination fiber bundles; 3 and 3': front and rear lenses; 4: telecentric slit aperture; 5 : mirror; 6 : detector light wave guides.

$=8 \mathrm{~mm}$. Because of the restricted space available for installing a sensor at a belt drive, the optical system contains a 45 deg mirror. This allows for a perpendicular view of the sensor on the belt surface even at places where two ends of the belt are guided close to one another.

The V-belt is illuminated by halogen light through two fiber optic bundles (see Fig. 9). The reflected radiation is collected by two $0.4-\mathrm{mm}$ glass light waveguides aligned at a distance of $L^{\prime}=6.3 \mathrm{~mm}$ from each other in the image plane of the optical system. They transmit the optical signals to two photodiodes in a signal processing unit.

\subsection{Intensity of the Signals}

To investigate the influence of the aperture size on the intensity of the detector signals, their magnitudes obtained with a circular and a rectangular aperture were compared. For this purpose, the sensor was placed over a still-standing white paper at the nominal distance of $o=20 \mathrm{~mm}$ between the paper and the front side of the first lens. The measured signal magnitudes are shown in Table 2. Using the rectangular aperture, a gain of 18.6 of the signal magnitude is obtained, compared to the value with the circular aperture. Thus the measured gain does not reach the theoretic value
Table 2 Measured signal magnitudes with circular and rectangular telecentric apertures.

\begin{tabular}{lcc}
\hline \hline Aperture & Circular & Rectangular \\
Dimensions & $0.4 \mathrm{~mm}$ & $0.4 \mathrm{~mm} \times 8 \mathrm{~mm}$ \\
Signal magnitude & $0.54 \mathrm{~V}$ & $9.95 \mathrm{~V}$ \\
Normalized & 1 & 18.6 \\
\hline \hline
\end{tabular}

of 25 (cf. Table 1), but it is of the same order of magnitude. The deviation from the theoretical value is probably due to the fact that the angle of incidence of the illuminating light on the paper is inclined and that the reflection on the paper surface is not exactly diffuse.

\subsection{Dependence of the Optical Magnification on the Object Distance}

\subsubsection{Direct measurement}

As already shown, the optical magnification $M$ of the sensor needs to be constant. To investigate whether this requirement is satisfied, $M$ can be measured in the following manner. The sensor is positioned at different distances in front of a test surface, while its light waveguides are illuminated from the detector side. Hence, the direction of the radiation passing through the optical system is inverted, enabling us to visualize the surface segments that would be imaged onto the detectors at normal operating conditions of the sensor.

Placing a camera opposite to the sensor behind a millimeter-scaled transparent paper, we obtained the photographs shown in Fig. 10. The bright areas are aligned at a distance of $L=L^{\prime} / M=4.2 \mathrm{~mm}$ from each other, as can be expected from the sensor's optical setup as described. This distance does not depend on whether or not the sensor is at the nominal distance from the transparent paper. Thus, the optical system is telecentric in the direction between the light waveguides, as is required for 1-D speed measurement.

The photographs of Fig. 10 show further that only in case of focused imaging, circular surface segments are imaged onto the circular end faces of the light waveguides. At nonzero deviations $\Delta o$ from the nominal distance $o$

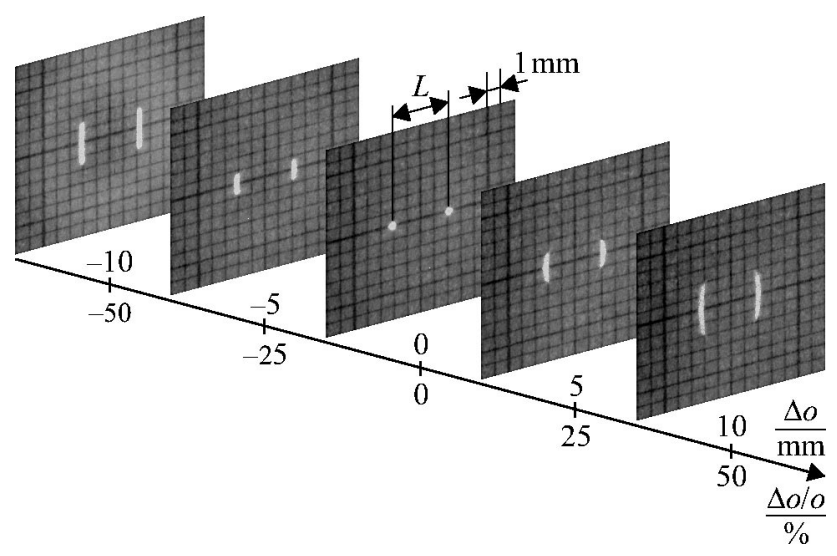

Fig. 10 Surface segments imaged onto the sensor's light waveguides at different object distances. 
$=20 \mathrm{~mm}$, the bright surface segments are blurred in the direction perpendicular to the telecentric direction. Due to the rectangular aperture of the system, the shape of the imaged surface segments is then approximately rectangular.*

Finally, Fig. 10 shows a distortion of the quasirectangular shape at positive deviations from the nominal sensor distance. The bright areas appear rather like segments of a ring, which results either from spherical aberration of the uncorrected lenses or from limitation of the imaged rays by the edges of the lenses.

\subsubsection{Indirect verification}

Alternatively to the method just described, errors of the optical magnification can be detected indirectly from speed measurement. Assuming a V-belt velocity $v$, the time lag $T^{\prime}=L^{\prime} /\left(M^{\prime} v\right)$ evaluated by correlation techniques differs from the theoretical value $T=L^{\prime} / M v$ [cf. Eq. (1)] if $M^{\prime}$ does not equal the nominal value $M$. Thus, calculating the measured velocity $v_{m}$ according to Eq. (1) leads to a relative measuring error of

$\frac{\Delta v}{v}=\frac{v_{m}-v}{v}=\frac{T-T^{\prime}}{T^{\prime}}=\frac{M^{\prime}-M}{M}=\frac{\Delta M}{M}$.

This equation shows that relative errors of the optical magnification lead to equivalent measurement errors of the velocity.

The disadvantage of the described method is that measurement errors of the velocity do not necessarily indicate magnification errors of the optical imaging system. They can also be due to unsymmetrical cross-correlation functions, ${ }^{10}$ which often result from inhomogeneous illumination. Thus Eq. (15) is valid only for a perfectly homogenous illumination of the V-belt.

The following experimental setup was chosen: The V-belt was driven at a constant velocity of $5 \mathrm{~m} / \mathrm{s}$ at a test rig, while the sensor was adjusted at distances between 15 and $25 \mathrm{~mm}$ above the belt surface. At each distance, the measured velocity $v_{m}$ was computed from an average of 500 measured values of the time lag $T^{\prime}$ evaluated by a digital correlator. ${ }^{7}$ A reference value $v$ of the velocity was obtained from 500 synchronously recorded measured values of the rotational speed.

Calculating the deviations $\Delta v=v_{m}-v$ yields the curve of Fig. 11. The measuring error of the velocity shows only a minor dependence on the distance of the sensor to the belt. The measuring accuracy of $|\Delta v / v| \leqslant 0.3 \%$ within a distance range of $10 \mathrm{~mm}$ is sufficient for the application on V-belts. As explained, the measurement error of the velocity does not necessarily correspond to deviations of the optical magnification, but may be due to distance-dependent variations of the illumination. Thus, the measurement accuracy could be further improved by optimizing the illumination of the V-belt with regard to homogeneity.

\footnotetext{
*The exact shape, which can be derived from Fourier optical analysis, is obtained from convoluting a rectangle with a circle.
}

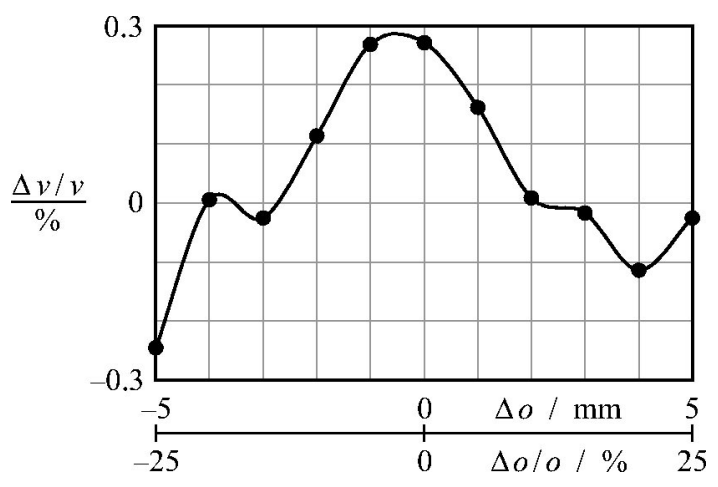

Fig. 11 Relative error of the measured velocity.

\section{Summary}

This paper elaborated on telecentric imaging systems for noncontact velocity sensors. Although their optical setup is quite simple, various design considerations must be taken into account when setting up custom systems for prototype sensors. Dimensioning of the aperture stop must balance two conflicting aims: A high tolerance toward variations of the object distance requires smallest possible aperture dimensions, whereas a high luminous intensity of the image is obtained only with large apertures. In the case of 1-D velocity sensors, rectangular aperture stops may help to better satisfy both requirements.

Inaccurate axial positioning of optical elements leads to an undesired dependence of the optical magnification on the object distance. As significant magnification errors may occur especially in systems with small nominal values of the optical magnification, the design of such systems should be avoided, whenever possible. Further, the sensitivity to axial misalignments is generally lower for double than for single telecentric systems.

The preceding considerations were realized in the design of an optical V-belt velocity sensor.

\section{References}

1. R. Zeitler and C. Berger, "Geschwindigkeitsmessung von Papierbahnen mit Korrelationsverfahren," Das Papier 51(6), 287-295 (1997).

2. R. Zeitler and S. Haap, "Längenmessung von Fasergarnen beim Spulproze $\beta$," Melliand Textilber. 77, 192-198 (1996).

3. C. Berger, "Optical sensors for strain measurement on moving V-belts," in Proc. 18th IEEE Instrumentation and Measurement Technology Conf., pp. 266-271, Budapest (2001).

4. C. Berger and A. Wehle, "Messung von Schlupf und Dehnung in Riementrieben," VDI-Ber. 1608, 863-870 (2001).

5. C. Berger, K.-H. Hanus, A. Wehle, and O. Wüstefeld, "Bestimmung des scheibenspezifischen Schlupfes in Kraftfahrzeug-Riementrieben," MTZ Motortechn. Zeitschr. 63(1), 46-51 (2002).

6. F. Mesch, "Speed and flow measurement by an intelligent correlation system," in Proc. ISA 90, pp. 1899-1914 New Orleans (1990).

7. R. Zeitler "Digital correlator for measuring the velocity of solid surfaces," IEEE Trans. Instrum. Meas. 46(4), 803-806 (1997).

8. A. Hayashi and Y. Kitagawa, "Image velocity sensing using an optical fiber array," Appl. Opt. 21(8), 1394-1399 (1982).

9. O. Fiedler, J. Werther, N. Labahn, J. Kumpart, and K. Christofori, "Measurement of local particle velocities and velocity distributions in gas-solid flows by means of the spatial filter method," Powder Technol. 94(1), 51-57 (1997).

10. R. Zeitler, "Digitale Korrelationsme $\beta$ systeme zur eindimensionalen Geschwindigkeitsmessung fester Oberflächen," $\mathrm{PhD}$ Thesis, Universität Karlsruhe (TH), VDI-Verlag, Düsseldorf (1998).

11. M. Watanabe and S. K. Nayar, "Telecentric optics for focus analysis,' IEEE Trans. Pattern Anal. Mach. Intell. 19(12), 1360-1365 (1997).

12. R. Schumann and T. Thöni $\beta$, "Telezentrische Systeme für die optische Me $\beta$ - und Prüftechnik," Tech. Mess. 65(4), 131-136 (1998).

13. R. Freimann and H. Gross, "Propagation of the phase distribution 
through double telecentric optical systems," Optik 105(2), 69-73 (1997).

14. J. W. Goodman, Introduction to Fourier Optics, McGraw-Hill, New York (1968)

15. J. D. Gaskill, Linear Systems, Fourier Transforms, and Optics, John Wiley \& Sons, New York (1978).

16. D. C. Champeney, Fourier Transforms and Their Physical Applications, Academic Press, London (1973)

17. K. Michel, O. Fiedler, A. Richter, K. Christofori, and S. Bergeler, "A novel spatial filtering velocimeter based on a photodetector array," IEEE Trans. Instrum. Meas. 47(1), 299-303 (1998).

18. M. Born and E. Wolf, Principles of Optics, 6th ed., Cambridge University Press, Cambridge (1998)

19. C. Hofmann, Die optische Abbildung, Akademische Verlagsgesellschaft Geest \& Portig K. G., Leipzig (1980).

20. F. Mesch, "Systemtheoretische Beschreibung optisch-elektrischer $\mathrm{Me} \beta$ systeme. Teil 1: Inkohärente Systeme mit beliebigen Blenden und mit Defokussierung-Einführung und Ubersicht," Tech. Mess. 44(78), 249-258 (1977).

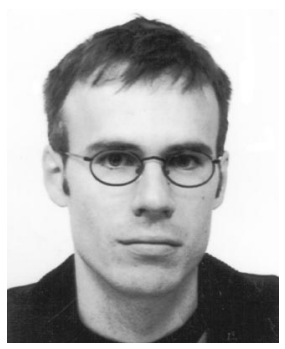

Christian Berger received his MS degree in mechanical engineering from the University of Karlsruhe, Germany, in 1997. Since 1998, he has been a research assistant with the Institut für Mess- und Regelungstechnik. His research interests include optical sensors for noncontact velocimetry and correlation methods. His current work focuses on optimization of the optical and electronic design of such sensors to avoid systematic measurement errors. 